\title{
Chlorogenic acid prevents isoproterenol-induced DNA damage in vascular smooth muscle cells
}

\author{
JINGSHUAI WANG $^{1^{*}}$, JIYANG LI $^{2 *}, \mathrm{JIE} \mathrm{LIU}^{3^{*}}$, MENGJIAO XU $^{4}$, XIAOWEN TONG ${ }^{4}$ and JIANJUN WANG ${ }^{4}$ \\ ${ }^{1}$ Department of Obstetrics and Gynecology, Shanghai First Maternity and Infant Hospital, Shanghai 201204; \\ ${ }^{2}$ Department of General Medicine, Shanghai Ninth People's Hospital, Shanghai Jiaotong University School of Medicine, \\ Shanghai 200000; ${ }^{3}$ Department of Cardiovascular and Thoracic Surgery, Shanghai East Hospital, \\ Tongji University School of Medicine, Shanghai 200120; ${ }^{4}$ Department of Obstetrics and Gynecology, \\ Tongji Hospital of Tongji University, Tongji University School of Medicine, \\ Shanghai 200065, P.R. China
}

Received September 29, 2015; Accepted June 30, 2016

DOI: $10.3892 / \mathrm{mmr} .2016 .5743$

\begin{abstract}
Numerous clinical therapeutic agents have been identified as DNA damaging. The present study revealed that isoproterenol (Iso) resulted in DNA damage in vascular smooth muscle cells (VSMCs) and increased the levels of intracellular oxygen free radicals. Administration of chlorogenic acid (CGA) inhibited this effect. Pretreatment with CGA abrogated the increase in protein expression levels of $\gamma$-H2A histone family member $\mathrm{X}$, phosphorylated ataxia telangiectasia mutated, phosphorylated Rad3-related protein, breast cancer 1 and C-terminal Src homologous kinase induced by Iso. In addition, the increase in levels of intracellular reactive oxygen species (ROS) induced by Iso was inhibited by CGA pretreatment in a dose-dependent manner. The results of the present study suggest that CGA may inhibit Iso-induced VSMC damage via the suppression of ROS generation. Therefore, CGA may be a novel agent for the treatment of vascular diseases.
\end{abstract}

\section{Introduction}

Various types of DNA lesions may result in genomic instability. To rectify this situation, cells activate the DNA damage response to recognize and repair DNA lesions (1). However, when complete repair of the damage is not achieved, the accumulation of unrepaired DNA damage may result in various diseases, including cancer (2), immunodeficiency (3)

Correspondence to: Professor Jianjun Wang, Department of Obstetrics and Gynecology, Tongji Hospital of Tongji University, Tongji University School of Medicine, 389 Xincun Road, Shanghai 200065, P.R. China

E-mail: jjwang0524@sina.com

*Contributed equally

Key words: chlorogenic acid, isoproterenol, DNA damage, reactive oxygen species and cardiovascular disease (4). The primary causes of DNA damage may be divided into exogenous and endogenous factors, the latter of which primarily arise due to intracellular oxidative stress. Free radicals are a class of substances containing unpaired electrons, of which oxygen free radicals account for $\sim 95 \%$. Free radicals are generated in three ways: i) Cracking of covalent bonds, ii) single-electron loss and iii) single-electron gain. On entering the body, a large proportion of oxygen is utilized by the respiratory chain, whereas $2-3 \%$ is catalyzed by oxidase and further converted to reactive oxygen species (ROS), the majority of which is eliminated by antioxidant enzymes and molecules $(1,5,6)$. However, excessive ROS, which may occur following the breakdown of the body homeostasis of oxidation and antioxidation, may attack double-stranded DNA and impair DNA repair mechanisms, resulting in DNA breakage and nucleotide modifications (7).

Atherosclerosis (AS) is a primary cause of mortality and morbidity in high-income countries (8). It is predicted that the mortality rate of atherosclerosis will surpass that of infectious diseases by 2020 (9). In recent years, studies on signal transduction and gene regulation have revealed that oxidative stress and inflammation are two key elements in AS occurrence. ROS and oxidized low density lipoprotein are the primary causes of endothelial injury and excessive production of inflammatory cytokines. In addition, by increasing growth factors such as platelet derived growth factor, ROS indirectly transform vascular smooth muscle cells (VSMCs) from a contractile to a synthetic phenotype. Synthetic phenotype VSMCs synthesize collagen and elastic fibers, producing a fibrous cap surrounding the lipid pool and forming a typical atherosclerotic plaque. As the fibrous cap ages, it becomes thinner and more prone to rupture (10). Matthews et al (11) revealed that oxidative stress may lead to DNA damage in VSMCs by the breakage of DNA strands, oxidative modification of guanine and regulation of associated enzyme activity, and ultimately affect the occurrence and progression of AS.

Numerous therapeutic agents have been demonstrated to stimulate DNA damage (12-14). Isoproterenol (Iso), a synthetic catecholamine and potent vasodilator, is widely administered in cardiac arrest and shock $(15,16)$. However, it has been 
demonstrated to induce DNA damage in cardiomyocytes and results in cardiac wall hypertrophy $(17,18)$. Kim et al (19) demonstrated that Iso elevated levels of intracellular ROS and increased cerebrovascular damage.

The present study aimed to assess the prevention of Iso-induced vascular damage via treatment with a compound present in various herbs. Chlorogenic acid (CGA) is a naturally occurring polyphenol, which is the main active ingredient in traditional Chinese medicine honeysuckle and eucommia leaves and is present in sunflower seeds, fruits, vegetables, soybeans, wheat and coffee beans. CGA has anticancer, anti-inflammatory and anti-oxidative activities (20-22). CGA is formed by the condensation of caffeic acid and quinic acid, two phenolic compounds. As it has phenolic hydroxide groups, CGA is highly reductive and extremely vulnerable to oxidation, enabling it to scavenge free radicals and affect anti-lipid peroxidation.

In the present study, Iso-induced DNA damage in VSMCs and the levels of intracellular ROS were analyzed, and the protective effect of CGA pretreatment on this damage was investigated.

\section{Materials and methods}

Reagents. Iso, CGA, collagenase II and trypsin were purchased from Sigma-Aldrich (St. Louis, MO, USA). Primary rabbit antibodies against phosphorylated (p) ataxia telangiectasia mutated (ATM; catalog no. 13050), p-Rad3-related protein (ATR; catalog no. 2853), p-breast cancer 1 (BRCA1; catalog no. 9009), p-C-terminal Src homologous kinase 2 (Chk2; catalog no. 2197), $\gamma$-H2A histone family member X $(\gamma$-H2AX; catalog no. 2595) and TATA binding protein (TBP; catalog no. 8515) were purchased from Cell Signaling Technology, Inc. (Danvers, MA, USA). Mouse anti- $\alpha$-smooth muscle actin ( $\alpha$-SMA; catalog no. A5228), which is a vascular-specific capture antibody, was obtained from Sigma-Aldrich. Rabbit anti-glyceraldehyde 3-phosphate dehydrogenase (GAPDH; catalog no. 5632-1) was purchased from Epitomics (Burlingame, CA, USA). Fluorescein isothiocyanate (FITC) -conjugated goat anti-mouse IgG (catalog no. sc-2010) was obtained from Santa Cruz Biotechnology, Inc. (Dallas, TX, USA). HRP-labeled goat anti-rabbit IgG (catalog no. A0208) and cell lysis buffer were purchased from Beyotime Institute of Biotechnology (Haimen, China). The bicinchoninic acid (BCA) protein quantitation kit was obtained from Pierce; Thermo Fisher Scientific, Inc. (Waltham, MA, USA). The SuperSignal chemiluminescence substrate kit was purchased from EMD Millipore (Billerica, MA, USA). All other chemicals used were of analytical grade.

Isolation and culture of VSMCs. A total of 22 C57BL/6 female mice was obtained from Shanghai Slac Laboratory Animal Co. Ltd. (Shanghai, China) and maintained in specific pathogen-free conditions at $22-26^{\circ} \mathrm{C}$ under a 12 -h light/dark cycle, with ad libitum access to food and water, at Tongji University (Shanghai, China). The protocol was approved by the Institutional Animal Care and Use Committee of Tongji University (Shanghai, China). The thoracic aorta was removed from 4- to 5-week-old mice following sacrifice by cervical dislocation. The aorta was digested with $1 \mathrm{mg} / \mathrm{ml}$ collagenase II

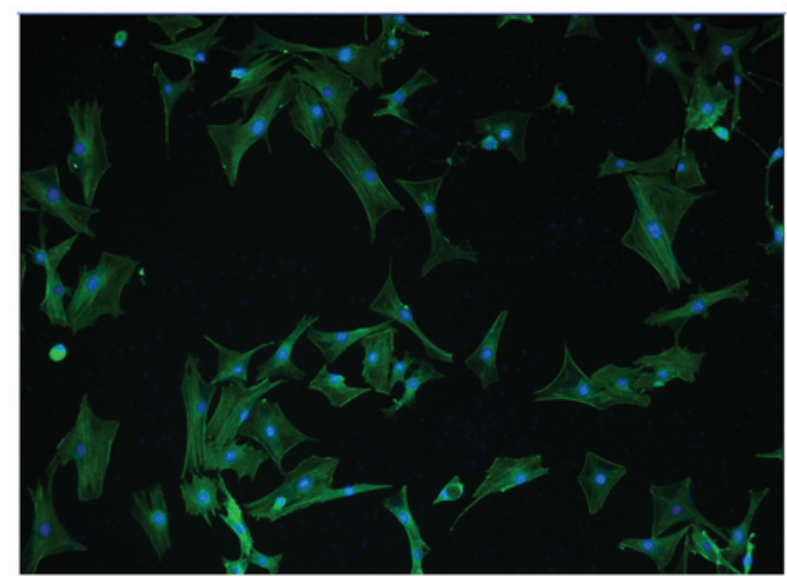

Figure 1. Immunofluorescence staining of isolated VSMCs. VSMCs were stained with an $\alpha$-SMA antibody and a fluorescein isothiocyanate-conjugated secondary antibody and counterstained with 4',6-diamidino-2-phenylindole. The majority of cells were positive for $\alpha$-SMA, and exhibited discernible fiber filaments and a well-formed spindle shape, verifying the high purity of isolated VSMCs. Magnification, x20. VSMCs, vascular smooth muscle cells; $\alpha$-SMA, smooth muscle $\alpha$-actin.

and $100 \mu \mathrm{g} / \mathrm{ml}$ trypsin at $37^{\circ} \mathrm{C}$ for $40 \mathrm{~min}$. Following digestion, cells were pelleted at 3,000 x $g$ for $6 \mathrm{~min}$ at room temperature, and plated in Dulbecco's modified Eagle's medium (DMEM; Wisent, Inc., St. Bruno, QC, Canada) containing 1 g/l glucose, $10 \%$ fetal bovine serum (Gibco; Thermo Fisher Scientific, Inc.) and $1 \%$ penicillin/streptomycin (Gibco; Thermo Fisher Scientific, Inc.). Cells were cultured in a humidified incubator with $5 \% \mathrm{CO}_{2}$ at $37^{\circ} \mathrm{C}$. Every $48 \mathrm{~h}$, cells were washed with phosphate-buffered saline (PBS) three times and placed in fresh media. Primary cells following the second passage were used for experiments.

Immunofluorescence identification of isolated cardiomyocytes. Cultured VSMCs were washed twice with PBS and fixed in 4\% paraformaldehyde solution (Sangon Biotech Co., Ltd., Shanghai, China) at room temperature for $20 \mathrm{~min}$. Following fixation, VSMCs were washed with PBS to remove the fixative and immersed in $0.5 \%(\mathrm{v} / \mathrm{v})$ Triton-X-100 (Sigma-Aldrich) in PBS for $5 \mathrm{~min}$ to achieve permeability. VSMCs were then washed with $0.5 \%$ Tween ${ }^{\circledR}$ 20 in PBS (PBST) three times and immersed in 5\% bovine serum albumin (BSA; Sigma-Aldrich) in PBS for $1 \mathrm{~h}$ at $37^{\circ} \mathrm{C}$. VSMCs were washed twice with PBST and incubated with the anti- $\alpha$-SMA antibody diluted 1:100 in 2\% BSA in PBS and covered with Parafilm overnight at $4{ }^{\circ} \mathrm{C}$. The primary antibody was removed via three washes with PBST. Subsequently, a FITC-conjugated secondary antibody at a dilution of 1:200 in $2 \%$ BSA was added and incubated at room temperature for $1 \mathrm{~h}$ in the dark. The secondary antibody was removed via washing three times in PBST for $5 \mathrm{~min}$, and cells were stained with 4',6-diamidino-2-phenylindole (DAPI) solution at room temperature in the dark for $20 \mathrm{~min}$. The staining solution was removed and the cells washed with PBST. Anti-fade mounting medium was added and cell imaging was performed under a fluorescence microscope (Olympus Corporation, Tokyo, Japan). Identification of VSMCs was confirmed by morphological observation. The majority of the 

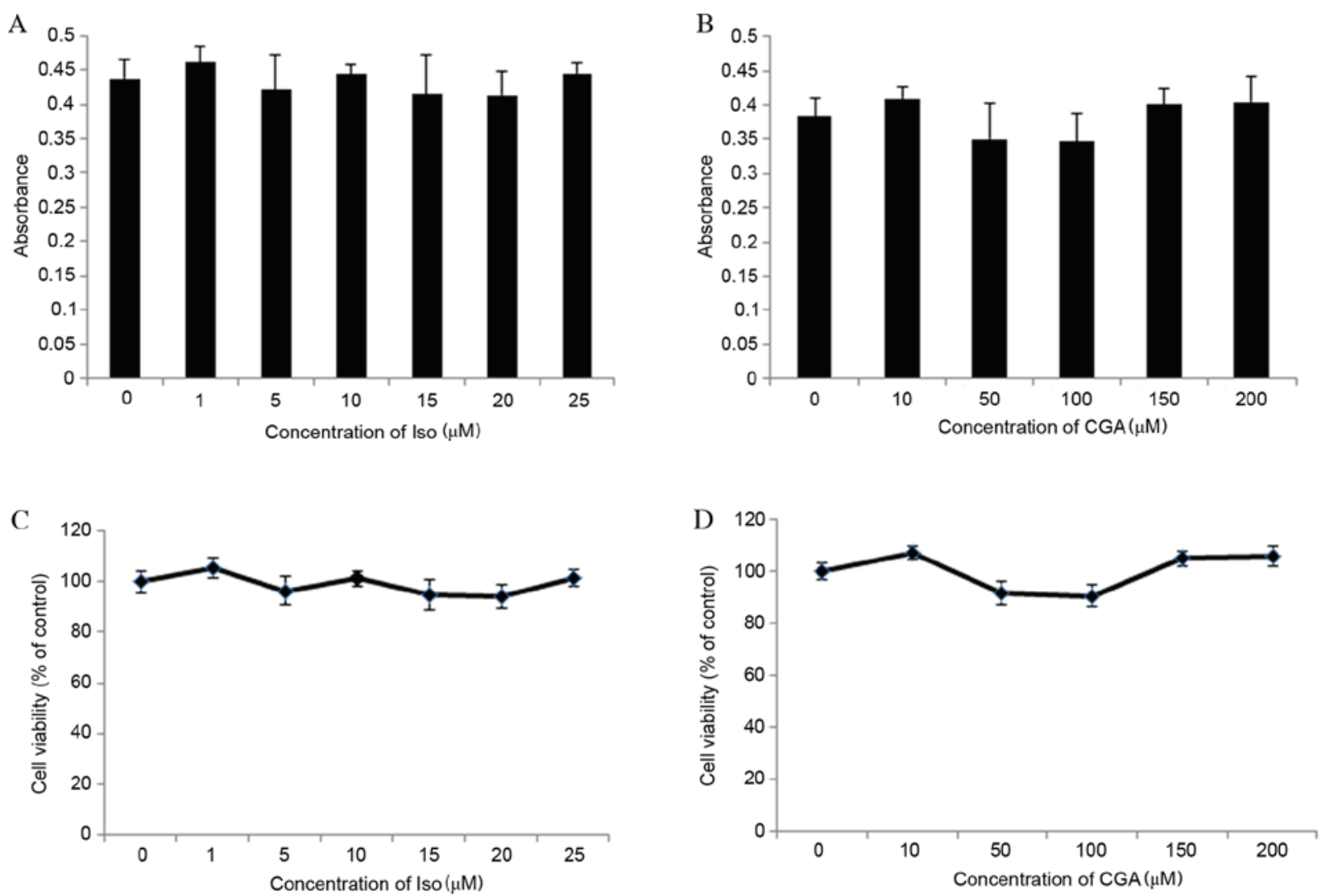

Figure 2. Effect of Iso and CGA on cytotoxicity and vascular smooth muscle cell viability. Cell viability was determined using an 3-(4,5-dimethylthiazol-2-yl)-2,5-diphenyltetrazolium bromide assay, which determined the absorbance of cells following treatment with various concentrations of (A) Iso and (B) CGA. The percentage of cell viability was calculated relative to control wells designated as $100 \%$ viable, for (C) Iso-and (D) CGA-treated cells. Iso, isoproterenol; CGA, chlorogenic acid.

VSMCs cultured in vitro exhibited a fusiform shape with a large nucleus and defined fiber filaments.

Cytotoxicity of Iso and CGA on VSMCs. Cell viability was measured by a 3-(4,5-dimethylthiazol-2-yl)-2,5-diphenyltetrazolium bromide (MTT) assay. Iso $(0,1,5,10,15,20$ or $25 \mu \mathrm{M})$ and CGA $(0,10,50,100,150$ or $200 \mu \mathrm{M})$ were added to a 96-well plate containing adhesive VSMCs (5,000 cells/well), with 6 wells for each concentration. Following incubation in a $5 \% \mathrm{CO}_{2}$ incubator at $37^{\circ} \mathrm{C}$ for $12 \mathrm{~h}$, the medium in each well was removed and the cells were washed twice with PBS. MTT solution (20 in $100 \mu 1 \mathrm{DMEM}$ ) was added to each well and the plate was incubated in a $5 \% \mathrm{CO}_{2}$ incubator at $37^{\circ} \mathrm{C}$ for $4 \mathrm{~h}$. The medium was removed and $150 \mu \mathrm{l}$ dimethyl sulfoxide (Sangon Biotech Co., Ltd.) was added to each well on a micro-oscillator (Kylin-Bell Lab Instruments Co., Ltd., Haimen, China); the plate was shaken for $10 \mathrm{~min}$ to dissolve the crystals. The absorbance in each well was read using a Multiskan FC microplate reader (Thermo Fisher Scientific, Inc.) at a wavelength of $570 \mathrm{~nm}$.

Western blot analysis. Following treatment, medium was removed and the cells were washed three times with PBS. Cells were lysed in cell lysis buffer containing $1 \%$ phenylmethylsulfonyl fluoride (PMSF; Beyotime Institute of Biotechnology) for $30 \mathrm{~min}$ on ice and centrifuged at $14,000 \times \mathrm{g}$ for $10 \mathrm{~min}$ at $4^{\circ} \mathrm{C}$. Nuclear proteins were extracted from Iso-treated cells using a nuclear and cytoplasmic protein extraction kit (Beyotime Institute of Biotechnology) containing $1 \%(\mathrm{v} / \mathrm{v})$ PMSF. The total protein contents were quantified with a BCA protein assay kit to ensure equal protein loading $(\sim 50 \mu \mathrm{g}$ protein) in each well prior to gel electrophoresis. The prepared samples were boiled in $5 \mathrm{X}$ sodium dodecyl sulfate-polyacrylamide gel electrophoresis (SDS-PAGE) sample loading buffer (Beyotime Institute of Biotechnology) for $5 \mathrm{~min}$. The samples were separated on $10 \%$ SDS-PAGE gels and transferred to polyvinylidene difluoride membranes. Membranes were blocked for $1 \mathrm{~h}$ in 5\% non-fat milk in Tris/HCl-buffered saline containing $1 \%$ Tween 20 and $0.8 \% \mathrm{NaCl}$ (TBST) at room temperature and then incubated overnight with appropriate primary antibodies at $4^{\circ} \mathrm{C}$. The dilution of all primary antibodies was 1:1,000, except the anti-GAPDH antibody, which was diluted to $1: 5,000$. Following washing with TBST, the membranes were incubated with the HRP-conjugated secondary antibody $(1: 3,000)$ for $1 \mathrm{~h}$ at room temperature. The membranes were rinsed and soaked in SuperSignal chemiluminescence substrate kit. Immunoreactive bands were visualized with a chemiluminescence imaging analyzer and Quantity One ${ }^{\circledR}$ software version 4.6.2 (Bio-Rad Laboratories, Inc., Hercules, CA, USA).

Measurement of ROS. The level of intracellular ROS following Iso and CGA treatment was measured using a 
A

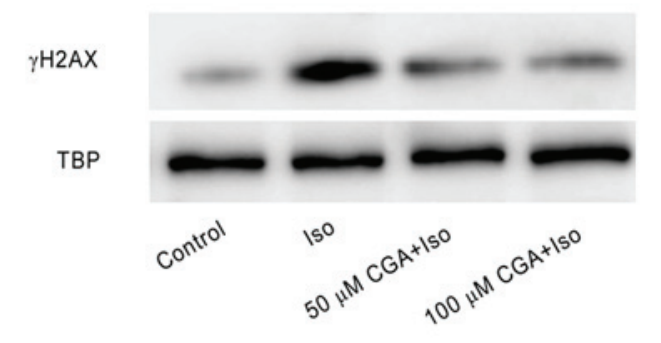

B

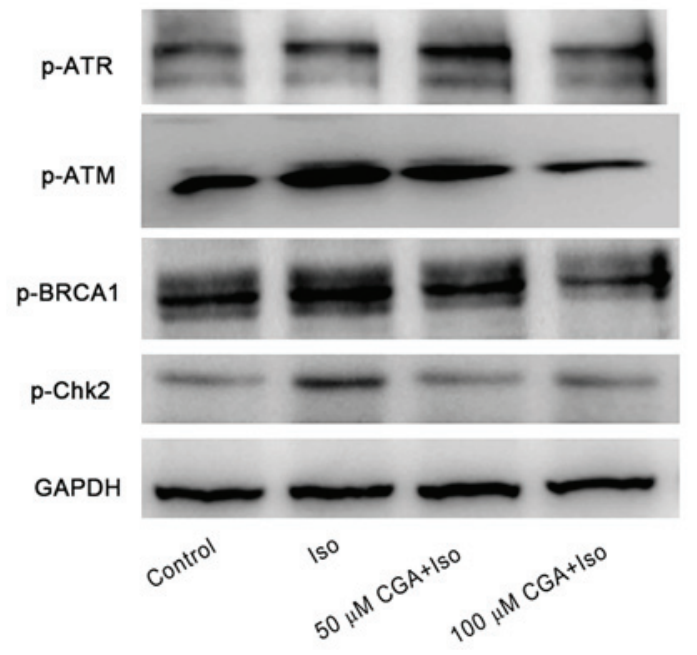

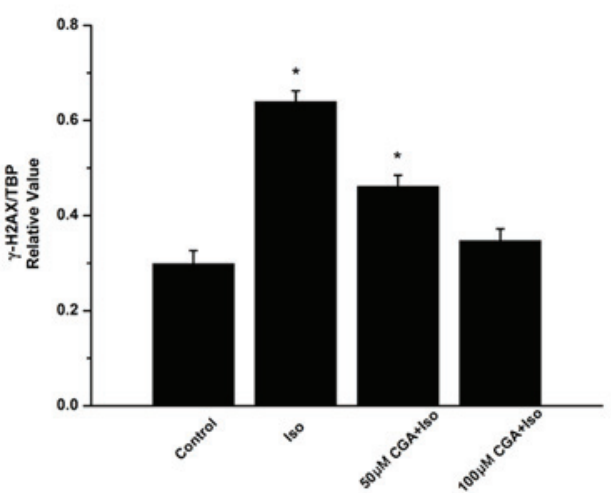

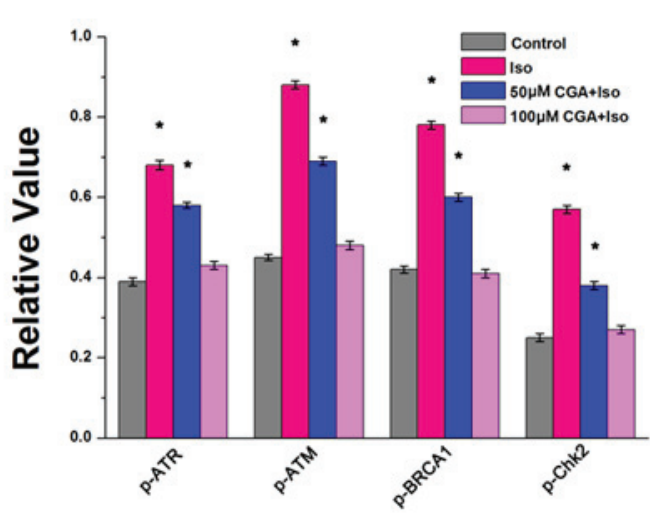

Figure 3. DNA damage following treatment of vascular smooth muscle cells with Iso, in the presence or absence of CGA pretreatment, as determined by western blot analysis. (A) Alterations in the protein expression levels of the nuclear protein $\gamma$-H2AX. TBP served as a loading control. (B) Alterations in the protein expression levels of p-ATR, p-ATM, BRCA-1 and Chk2. GAPDH served as a loading control. The western blot represents one of at least three independent experiments that demonstrated similar results. ${ }^{*} \mathrm{P}<0.05$ vs. control. Iso, isoproterenol; CGA, chlorogenic acid; $\gamma$-H2AX, $\gamma$-H2A histone family member X; TBP, TATA binding protein; p, phosphorylated; ATR, Rad3-related protein; ATM, ataxia telangiectasia mutated; BRCA1, breast cancer 1; Chk2, C-terminal Src homologous kinase 2; GAPDH, glyceraldehyde 3-phosphate dehydrogenase.

fluorescent probe, 2',7'-dichloro-dihydro-fluorescein diacetate (DCFH-DA; Reactive Oxygen Species assay kit; Beyotime Institute of Biotechnology), as described previously (23). In brief, cells were loaded with serum-free medium containing $10 \mu \mathrm{M}$ DCFH-DA (v/v, 1:1,000) and incubated at $37^{\circ} \mathrm{C}$ for $30 \mathrm{~min}$. The residual DCFH-DA solution was removed and cells were washed three times with serum-free medium for $5 \mathrm{~min}$, and then with PBS. Cells were observed under a fluorescence microscope (Olympus Corporation) equipped with a charge-coupled device camera (Hamamatsu Photonics K.K., Hamamatsu, Japan). Quantification of the fluorescence intensity was performed using ImageJ software version 1.42 (National Institutes of Health, Bethesda, MD, USA). The data are expressed as percentages compared with the control intensity.

Statistical analysis. Statistical analyses were performed using SPSS software version 14.0 (SPSS, Inc., Chicago, IL, USA). Groups were compared using one-way analyses of variance and Fisher's protected least significant difference test. All data are expressed as the mean \pm standard deviation. $\mathrm{P}<0.05$ was considered to indicate a statistically significant difference. The experiments were repeated at least three times independently.

\section{Results}

High-purity isolation of VSMCs. Immunofluorescence staining using an $\alpha$-SMA antibody and a FITC-conjugated secondary antibody was performed to determine whether the isolated cells were high-purity VSMCs. Cells were counterstained with DAPI, which penetrates the cell membrane and binds to double-stranded DNA, fluorescing blue. $\alpha$-SMA positive cells accounted for $>95 \%$ of total cells from at least five fields of view, indicating the high purity of VSMCs (Fig. 1). In addition, VSMCs isolated following $48 \mathrm{~h}$ in culture exhibited discernible fiber filaments and a well-formed spindle shape.

Effects of Iso and CGA on cell viability. Cell viability was determined using an MTT assay, which determined the optical density (OD) values of Iso-and CGA-treated cells to be between 0.3 and 0.5 (Fig. 2A and $\mathrm{B}$, respectively). OD values between 0 and 0.7 are consistent with the linear association of cell viability. The percentage of cell viability was calculated relative to control wells designated as $100 \%$ viable. The results revealed that concentrations of Iso ranging from 1 to $25 \mu \mathrm{M}$ and CGA ranging from 10 to $200 \mu \mathrm{M}$ did not significantly affect cell viability compared with the control (Fig. 2C and D, respectively). Therefore, the effects of Iso 
A

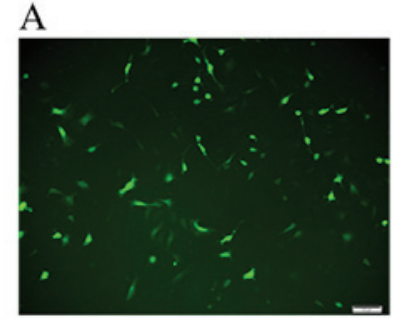

B

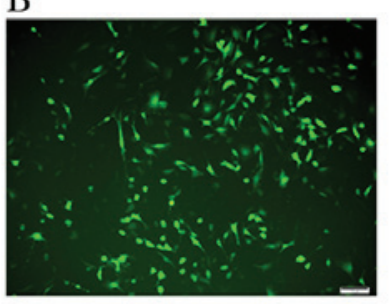

$\mathrm{C}$

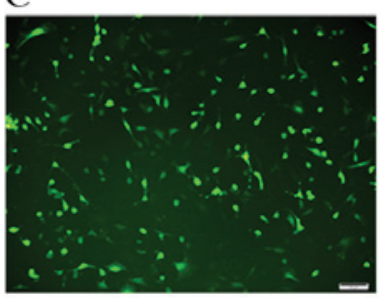

$\mathrm{D}$

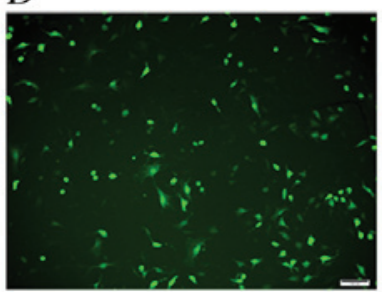

$\mathrm{E}$

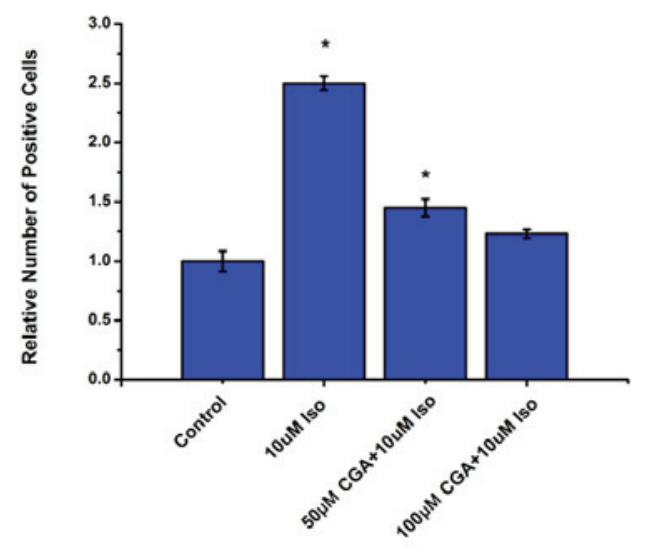

Figure 4. Levels of ROS in vascular smooth muscle cells following treatment with Iso, in the absence or presence of CGA pretreatment, as measured using a fluorescent probe, 2',7'-dichloro-dihydro-fluorescein diacetate. ROS levels were measured in (A) control cells, (B) $10 \mu \mathrm{M}$ Iso-treated cells, (C) $50 \mu \mathrm{M}$ CGA-and $10 \mu \mathrm{M}$ Iso-treated cells, and (D) $100 \mu \mathrm{M}$ CGA- and $10 \mu \mathrm{M}$ Iso-treated cells. (E) Quantification ROS levels. Scale bars $=100 \mu \mathrm{M}$. ${ }^{*} \mathrm{P}<0.05$ vs. control. ROS, reactive oxygen species; Iso, isoproterenol; CGA, chlorogenic acid.

and CGA observed in subsequent experiments were not due to cell toxicity.

CGA protects VSMCs from Iso-induced DNA damage. $\gamma$-H2AX, a double-stranded DNA break marker of DNA damage, is expressed in the nuclei of cells. TBP served as the loading control for nuclear proteins. Following treatment of VSMCs with $10 \mu \mathrm{M}$ Iso for $48 \mathrm{~h}$, the protein expression level of $\gamma$-H2AX increased significantly by 2.1 -fold $(\mathrm{P}=0.0012$; Fig. 3A). However, pretreatment with $50 \mu \mathrm{M}$ CGA significantly inhibited Iso-induced $\gamma-\mathrm{H} 2 \mathrm{AX}$ upregulation by $50 \%$ compared with the Iso group without CGA pretreatment $(\mathrm{P}=0.0081)$. Furthermore, almost no change in $\gamma$-H2AX was visible compared to the control group following $100 \mu \mathrm{M}$ CGA pretreatment of the Iso-induced VSMCs $(\mathrm{P}=0.1050)$. These results indicated that CGA may inhibit Iso-induced DNA strand breaks.

To investigate the signaling pathway of Iso-induced DNA damage, alterations in the protein expression levels of DNA damage-associated proteins were observed. There were similar trends in the protein expression levels of p-ATM, p-ATR, p-BRCA1 and p-Chk2, which reflected changes in $\gamma-\mathrm{H} 2 \mathrm{AX}$ expression. Iso treatment induced significant upregulation of these DNA damage associated proteins relative to the control (p-ATM, $\mathrm{P}=0.0023$; p-ATR, $\mathrm{P}=0.0015$; p-BRCA1, $\mathrm{P}=0.0013$; $\mathrm{p}-\mathrm{Chk} 2, \mathrm{P}=0.0020)$. Following CGA pretreatment, Iso-induced DNA damage was notably reduced in VSMCs (Fig. 3B). The inhibition of DNA damage-induced upregulation of p-ATM, p-ATR, p-BRCA1 and p-Chk2 expression levels was CGA dose-dependent. These results suggested that CGA may inhibit Iso-induced DNA damage checkpoint arrest.

$C G A$ reduces Iso-induced $R O S$ in VSMCs. It has previously been reported that there is an association between inflammation and ROS $(24,25)$, and that CGA scavenges oxygen free radicals in cells $(26,27)$. Therefore, ROS levels were examined in VSMCs treated with Iso in the absence or presence of CGA treatment. Following Iso treatment, the level of ROS significantly increased in VSMCs after $48 \mathrm{~h}(\mathrm{P}=0.0008)$. Pretreatment with CGA significantly decreased the level of ROS compared with Iso treatment alone $(50 \mu \mathrm{M}, \mathrm{P}=0.0327$; $100 \mu \mathrm{M}, 0.0938$; Fig. 4). At $10 \mu \mathrm{M}$ CGA, the ROS level was reduced from an increase of 2.3-fold to an increase of 1.6-fold relative to untreated cells. Furthermore, $50 \mu \mathrm{M}$ CGA resulted in a further decrease of ROS levels. At $100 \mu \mathrm{M}$ CGA, the level of ROS in VSMCs returned almost to the basal level of control cells (Fig. 4). These results indicated that the level of ROS increased during Iso-induced DNA damage and that CGA may inhibit DNA damage by suppressing ROS.

\section{Discussion}

VSMCs are crucial for maintaining the physiological function and remodeling of blood vessels $(28,29)$. Oxidative stress leading to cell DNA damage can accelerate the aging process in VSMCs, known as stress-induced premature senescence, which plays a crucial role in the process of the formation of AS (30). Therefore, investigation into the effects of Iso on VSMCs and the prevention of DNA damage induced by this therapeutic agent is key. CGA, as an active component of certain Chinese herbal medicines, has been investigated due to its various effects, including anticancer and anti-inflammatory effects. Pang et al (31) revealed that CGA may prevent acetaminophen-induced liver oxidative stress injury via the regulation of cytochrome $\mathrm{P} 450$ metabolism enzymes and certain important anti-oxidant signal molecules, including proteins from the peroxiredoxins family. Cha et al (32) demonstrated that CGA reduced UVB-mediated oxidative stress in human HaCaT keratinocytes. The preventive effect of CGA with regards to VSMC damage and inhibition of Iso-induced DNA damage may have important implications in the clinical setting.

The present study revealed that $10 \mu \mathrm{M}$ Iso may induce DNA damage in VSMCs and increase intracellular ROS. Pretreatment with CGA, particularly at the $100 \mu \mathrm{M}$ concentration, may effectively block damage to VSMCs induced by Iso through abrogating the increase in protein expression levels of $\gamma$-H2AX, p-ATM, p-ATR, p-BRCA1 and p-Chk2 and further preventing ROS formation. In addition, the increase in levels of intracellular reactive oxygen species 
(ROS) induced by Iso was inhibited by CGA pretreatment in a dose-dependent manner. These results support the findings of previous studies $(22,33,34)$, suggesting that CGA may be a promising drug for protection against vascular diseases. Furthermore, CGA is widely present in plant leaves, flowers and fruits $(35,36)$, which are used as herbal ingredients in certain traditional Chinese medicines. Therefore, CGA may have potential applications for the prevention of DNA damage.

\section{Acknowledgements}

The authors would like to thank the Laboratory Animal Center of Tongji University (Shanghai, China) for their care of the mice.

\section{References}

1. Zhang C, Luo T, Cui S, Gu Y, Bian C, Chen Y, Yu X and Wang Z: Poly(ADP-ribose) protects vascular smooth muscle cells from oxidative DNA damage. BMB Rep 48: 354-359, 2015.

2. Lord CJ and Ashworth A: The DNA damage response and cancer therapy. Nature 481: 287-294, 2012.

3. Gasser S and Raulet D: The DNA damage response, immunity and cancer. Semin Cancer Biol 16: 344-347, 2006.

4. Lee SH and Blair IA: Oxidative DNA damage and cardiovascular disease. Trends Cardiovasc Med 11: 148-155, 2001.

5. Narayanaswamy PB, Hodjat M, Haller H, Dumler I and Kiyan Y: Loss of urokinase receptor sensitizes cells to DNA damage and delays DNA repair. PLoS One 9: e101529, 2014.

6. Valko M, Jomova K, Rhodes CJ, Kuča K and Musílek K: Redoxand non-redox-metal-induced formation of free radicals and their role in human disease. Arch Toxicol 90: 1-37, 2016.

7. Gray K, Kumar S, Figg N, Harrison J, Baker L, Mercer J, Littlewood T and Bennett M: Effects of DNA damage in smooth muscle cells in atherosclerosis. Circ Res 116: 816-826, 2015.

8. Weber C and Noels H: Atherosclerosis: Current pathogenesis and therapeutic options. Nat Med 17: 1410-1422, 2011.

9. Fryburg DA and Vassileva MT: Atherosclerosis drug development in jeopardy: The need for predictive biomarkers of treatment response. Science Transl 3: 72cm6, 2011.

10. Kunieda T, Minamino T, Nishi J, Tateno K, Oyama T, Katsuno T, Miyauchi H, Orimo M, Okada S, Takamura M, et al: Angiotensin II induces premature senescence of vascular smooth muscle cells and accelerates the development of atherosclerosis via a p21-dependent pathway. Circulation 114: 953-960, 2006.

11. Matthews C, Gorenne I, Scott S, Figg N, Kirkpatrick P, Ritchie A, Goddard $\mathrm{M}$ and Bennett $\mathrm{M}$ : Vascular smooth muscle cells undergo telomere-based senescence in human atherosclerosis: Effects of telomerase and oxidative stress. Circ Res 99: 156-164, 2006.

12. Lebwohl M, Ting PT and Koo JY: Psoriasis treatment: Traditional therapy. Ann Rheum Dis (64 Suppl 2) ii83-ii86, 2005.

13. Havelka AM, Berndtsson M, Olofsson MH, Shoshan MC and Linder S: Mechanisms of action of DNA-damaging anticancer drugs in treatment of carcinomas: Is acute apoptosis an 'off-target' effect? Mini Rev Med Chem 7: 1035-1039, 2007.

14. Cheung-Ong K, Giaever G and Nislow C: DNA-damaging agents in cancer chemotherapy: Serendipity and chemical biology. Chem Biol 20: 648-659, 2013.

15. Leone M, Boyadjiev I, Boulos E, Antonini F, Visintini P, Albanèse $J$ and Martin C: A reappraisal of isoproterenol in goal-directed therapy of septic shock. Shock 26: 353-357, 2006.

16. Wiramus S, Textoris J, Bardin R, Vigne C, Kelway C, Martin C and Leone M: Isoproterenol infusion and microcirculation in septic shock. Heart Lung Vessel 6: 274-279, 2014.

17. Hara MR, Kovacs JJ, Whalen EJ, Rajagopal S, Strachan RT, Grant W, Towers AJ, Williams B, Lam CM, Xiao K, et al: A stress response pathway regulates DNA damage through $\beta 2$-adrenoreceptors and $\beta$-arrestin-1. Nature 477: 349-353, 2011.
18. Parthasarathy A, Gopi V, Devi K M S, Balaji N and Vellaichamy E: Aminoguanidine inhibits ventricular fibrosis and remodeling process in isoproterenol-induced hypertrophied rat hearts by suppressing ROS and MMPs. Life Sci 118: 15-26, 2014.

19. Kim HK, Park WS, Warda M, Park SY, Ko EA, Kim MH, Jeong SH, Heo HJ, Choi TH, Hwang YW, et al: Beta adrenergic overstimulation impaired vascular contractility via actin-cytoskeleton disorganization in rabbit cerebral artery. PLoS One 7: e43884, 2012

20. Feng R, Lu Y, Bowman LL, Qian Y, Castranova V and Ding M: Inhibition of activator protein-1, NF-kappaB, and MAPKs and induction of phase 2 detoxifying enzyme activity by chlorogenic acid. J Biol Chem 280: 27888-27895, 2005.

21. Yun N, Kang JW and Lee SM: Protective effects of chlorogenic acid against ischemia/reperfusion injury in rat liver: Molecular evidence of its antioxidant and anti-inflammatory properties. J Nutr Biochem 23: 1249-1255, 2012.

22. Li Y, Shen D, Tang X, Li X, Wo D, Yan H, Song R, Feng J, Li P, Zhang J and Li J: Chlorogenic acid prevents isoproterenol-induced hypertrophy in neonatal rat myocytes. Toxicol Lett 226: 257-263, 2014.

23. Degli Esposti M: Measuring mitochondrial reactive oxygen species. Methods 26: 335-340, 2002

24. Choudhury S, Ghosh S, Gupta P, Mukherjee S and Chattopadhyay S: Inflammation-induced ROS generation causes pancreatic cell death through modulation of Nrf2/NF- $\kappa \mathrm{B}$ and SAPK/JNK pathway. Free Radic Res 49: 1371-1383, 2015.

25. Kim W, Youn H, Kang C and Youn B: Inflammation-induced radioresistance is mediated by ROS-dependent inactivation of protein phosphatase 1 in non-small cell lung cancer cells. Apoptosis 20: 1242-1252, 2015.

26. Li Y, Shen D, Tang X, Li X, Wo D, Yan H, Song R, Feng J, Li P, Zhang J and Li J: Chlorogenic acid prevents isoproterenol-induced hypertrophy in neonatal rat myocytes. Toxicol Lett 226: 257-263, 2014.

27. Shi H, Shi A, Dong L, Lu X, Wang Y, Zhao J, Dai F and Guo X: Chlorogenic acid protects against liver fibrosis in vivo and in vitro through inhibition of oxidative stress. Clin Nutr S0261-5614: 00093-00095, 2016

28. Pruett ND, Hajdu Z, Zhang J, Visconti RP, Kern MJ, Wellik DM, Majesky MW and Awgulewitsch A: Changing topographic Hox expression in blood vessels results in regionally distinct vessel wall remodeling. Biol Open 1: 430-435, 2012.

29. Chen J, Xu L and Huang C: DHEA inhibits vascular remodeling following arterial injury: A possible role in suppression of inflammation and oxidative stress derived from vascular smooth muscle cells. Mol Cell Biochem 388: 75-84, 2014.

30. Kurz DJ, Decary S, Hong Y, Trivier E, Akhmedov A and Erusalimsky JD: Chronic oxidative stress compromises telomere integrity and accelerates the onset of senescence in humanendothelial cells. J Cell Sci 117: 2417-2426, 2004.

31. Pang C, Sheng YC, Jiang P, Wei H and Ji LL: Chlorogenic acid prevents acetaminophen-induced liver injury: The involvement of CYP450 metabolic enzymes and some antioxidant signals. J Zhejiang Univ Sci B 16: 602-610, 2015.

32. Cha JW, Piao MJ, Kim KC, Yao CW, Zheng J, Kim SM, Hyun CL, Ahn YS and Hyun JW: The polyphenol chlorogenic acid attenuates UVB-mediated oxidative stress in human HaCaT keratinocytes. Biomol Ther 22: 136-142, 2014.

33. Cinkilic N, Cetintas SK, Zorlu T, Vatan O, Yilmaz D, Cavas T, Tunc S, Ozkan L and Bilaloglu R: Radioprotection by two phenolic compounds: Chlorogenic and quinic acid, on X-ray induced DNA damage in human blood lymphocytes in vitro. Food Chem Toxicol 53: 359-363, 2013.

34. Wang Y, Zhang X, Zhang Q and Yang Z: Oxidative damage to DNA by 1,10-phenanthroline/L: -Threonine copper (II) complexes with chlorogenic acid. Biometals 23: 265-273, 2010.

35. Chen X, Sang X,Li S, Zhang S and Bai L: Studies on a chlorogenic acid-producing endophytic fungi isolated from Eucommia ulmoides Oliver. J Ind Microbiol Biotechnol 37: 447-454, 2010.

36. Yuan Y, Wang Z, Jiang C, Wang X and Huang L: Exploiting genes and functional diversity of chlorogenic acid and luteolin biosyntheses in Lonicera japonica and their substitutes. Gene 534: 408-416, 2014. 